\title{
TERMS IN CYLINDRIC ALGEBRAS ${ }^{1}$
}

\author{
CHARLES PINTER
}

\begin{abstract}
A new algebraic treatment of terms within the framework of cylindric algebras.
\end{abstract}

The theory of terms in locally finite polyadic algebras of infinite degree has been developed by Paul Halmos [2] and Aubert Daigneault [1]. In this paper we present a simple, new treatment of terms in dimension complemented cylindric algebras of infinite degree. By taking advantage of the presence of diagonal elements (which cannot be assumed to exist in arbitrary polyadic algebras), and by exploiting the well-known correspondence between operations and predicates which are single-valued in one of their variable places, we are able to introduce terms in a manner which is direct, well motivated and easy to use.

1. Introduction. Our notation and terminology will be that of Henkin, Monk and Tarski [3]. We begin with a brief discussion which is intended to motivate our definition of terms in cylindric algebras. This discussion is confined to the case of locally finite cylindric algebras, but the notions we are about to introduce are immediately extended, in the next section, to dimension complemented cyclindric algebras.

Two results from the literature will be useful to us here:

(A) If $\Lambda$ is a first order language, $\mathscr{F}_{m}(\Lambda)$ the free algebra of formulas in $\Lambda$, and $\theta$ a theory in $\Lambda$, then $\mathscr{F} m(\Lambda) / \equiv_{\theta}$ is a locally finite cylindric algebra called the cylindric algebra of formulas associated with $\theta$; conversely, every locally finite cylindric algebra of infinite degree is isomorphic to the cylindric algebra of formulas associated with some theory. ${ }^{2}$

(B) If $\theta_{1}$ and $\theta_{2}$ are first order theories, then the cylindric algebras associated with $\theta_{1}$ and $\theta_{2}$, respectively, are isomorphic iff $\theta_{1}$ and $\theta_{2}$ are definitionally equivalent. ${ }^{3}$

Received by the editors August 10, 1972.

AMS (MOS) subject classifications (1970). Primary 02J15.

Key words and phrases. Cylindric algebra, algebraic logic, terms.

${ }^{1}$ The work reported in this paper was done while the author held an NSF Faculty Fellowship.

${ }^{2}$ See $[3,1.1 .10,1.1 .11$, and the discussion on page 149].

${ }^{3}$ See $[4$, Theorem 1].

(c) American Mathematical Society 1973 
If $\mathfrak{A}$ is a locally finite cylindric algebra of infinite degree, then, by (A) above, there is a first order theory $\theta$ such that $\mathfrak{A}$ is isomorphic to the cylindric algebra of formulas of $\theta$. Let $\Lambda$ be the language of $\theta$.

A formula $P$ of $\Lambda$ is said to be functional in $v_{\kappa}$ (with respect to $\theta$ ) if $\theta \vdash\left(\exists ! v_{\kappa}\right) P$. For example, if $a$ is a term of $\Lambda$ and $v_{\kappa}$ does not occur in $a$, then the formula $v_{\kappa}=a$ is functional in $v_{\kappa}$. Conversely, if $P$ is any formula of $\Lambda$ which is functional in $v_{\kappa}$, then there is a theory $\theta^{\prime}$ which is an extension by definitions $s^{4}$ of $\theta$, and there is a term $a$ of the language of $\theta^{\prime}$, such that $P \equiv \equiv_{\theta^{\prime}}\left(v_{\kappa}=a\right)$. Indeed, if no variables other than $v_{\lambda_{1}}, \cdots, v_{\lambda_{n}}, v_{\kappa}$ are free in $P$, let $\theta^{\prime}$ be formed from $\theta$ by adding a new $n$-ary operation symbol $f$ and a new nonlogical axiom

$$
P \leftrightarrow v_{\kappa}=f v_{\lambda_{1}} \cdots v_{\lambda_{n}} ;
$$

if we let $a$ designate $f v_{\lambda_{1}} \cdots v_{\lambda_{n}}$, then $P \equiv_{\theta^{\prime}}\left(v_{\kappa}=a\right)$.

More generally, one easily shows that there is an extension by definitions $\theta^{*}$ of $\theta$ such that, if $P$ is any formula of the language of $\theta^{*}$ which is functional in some variable $v_{\kappa}$, then there is a term $a$ of the language of $\theta^{*}$ such that $P \equiv_{\theta^{*}}\left(v_{\kappa}=a\right)$. Clearly $\theta^{*}$ is definitionally equivalent to $\theta$, hence, by (B) above, $\mathfrak{A}$ is isomorphic with the cylindric algebra of formulas of $\theta^{*}$. Thus, there is no loss of generality if we assume that $\theta$ was chosen with the property that

(C) if $P$ is any formula of $\Lambda$ such that $\theta+\left(\exists ! v_{\kappa}\right) P$, then there is a term $a$ of $\Lambda$ such that $P \equiv_{\theta}\left(v_{\kappa}=a\right)$.

Now let us consider ordered pairs $\left(P, v_{\kappa}\right)$, where $P$ is a formula of $\Lambda, v_{\kappa}$ is a variable, and $P$ is functional in $v_{\kappa}$. If $\left(P, v_{\kappa}\right)$ is such a pair, then by $(C)$, there is a term $a$ of $\Lambda$ such that $P \equiv_{\theta}\left(v_{\kappa}=a\right)$; we say that the pair $\left(P, v_{\kappa}\right)$ determines $a$. [For any formula $Q$, let $S_{\mu}^{\kappa} Q$ designate the formula which results from $Q$ by validly replacing each free occurrence of $v_{\kappa}$ by $v_{\mu}$.] If $\left(P, v_{\kappa}\right)$ determines $a$, that is, $P \equiv_{\theta}\left(v_{\kappa}=a\right)$, and if $v_{\mu}$ does not occur in $a$, then $S_{\mu}^{\kappa} P \equiv_{\theta}\left(v_{\mu}=a\right)$; thus, $\left(S_{\mu}^{\kappa} P, v_{\mu}\right)$ determines $a$. Conversely, if $\left(P, v_{\kappa}\right)$ and $\left(Q, v_{\mu}\right)$ both determine $a$, then $P \equiv_{\theta}\left(v_{\kappa}=a\right)$ and $Q \equiv_{\theta}\left(v_{\mu}=a\right)$, hence $P \equiv_{\theta} S_{\kappa}^{\mu} Q$ and $Q \equiv_{\theta} S_{\mu}^{\kappa} P$. We may conclude as follows: $\left(P, v_{\kappa}\right)$ and $\left(Q, v_{\mu}\right)$ determine the same term iff $Q \equiv_{\theta} S_{\mu}^{\kappa} P$ and $P \equiv_{\theta} S_{\kappa}^{\mu} Q$.

The preceding remarks explain our definition of terms in cylindric algebras, which follows next. Roughly speaking, each term $a$ may be identified with the class of pairs $\left(P, v_{\kappa}\right)$ which determine $a$; that is, with a class of pairs $\left(P, v_{\kappa}\right)$ under the equivalence relation $\left(P, v_{\kappa}\right) \simeq\left(Q, v_{\mu}\right)$ iff $Q \equiv{ }_{\theta} S_{\mu}^{\kappa} P$ and $P \equiv{ }_{\theta} S_{\kappa}^{\mu} Q$.

2. The set of terms of a cylindric algebra. We come now to our main definition.

${ }^{4}$ See $[5$, p. 60]. 
(2.1) Definition. Let $\mathfrak{A}=\left\langle A,+, \cdot,-, 0,1, c_{\kappa}, d_{\kappa \lambda}\right\rangle_{\kappa, \lambda<\alpha}$ be a dimension complemented cylindric algebra of degree $\alpha \geqq \omega$. Let

$D=\left\{(x, \kappa): x \in A, \kappa<\alpha, c_{\kappa} x=1\right.$,

$$
\text { and for all } \left.\mu \in \alpha-\Delta x, x \cdot s_{\mu}^{x} x \leqq d_{\kappa \mu}\right\} \text {. }
$$

Let $\simeq$ designate the equivalence relation in $D$ given by $(x, \kappa) \simeq(y, \lambda)$ iff $y=s_{\lambda}^{\kappa} x$ and $x=s_{\kappa}^{\lambda} y$. Then $\mathscr{T}=D / \simeq$ is the set of all the terms of $\mathfrak{A}$.

In the remainder of this paper, let $\mathfrak{A}$ designate the dimension complemented cylindric algebra $\left\langle A,+, \cdot,-, 0,1, c_{\kappa}, d_{\kappa \lambda}\right\rangle_{\kappa, \lambda<\alpha}$ of degree $\alpha \geqq \omega$.

Let $a$ be a term of $\mathfrak{A}$; if $(x, \kappa) \in a$ and $\Delta x$ is the dimension set of $x$ [3, Definition 1.6.1], we let $\Delta a$ designate the set of ordinals

$$
\Delta a=\Delta x-\{\kappa\} .
$$

We call $\Delta a$ the dimension set of the term $a$; it follows immediately from $[3,1.6 .15)]$ that $\Delta a$ does not depend on the choice of a representative $(x, \kappa)$ in $a$. By $(2.2)$ together with [3, 1.5.4(i), 1.5.9(i) and 1.5.10(iv)-(vi)] one easily shows that

$$
\text { if }(x, \kappa) \in a \text { and } \lambda \in \alpha-\Delta a \text {, then }\left(s_{\lambda}^{\kappa} x, \lambda\right) \in a .
$$

Let $a$ be a term of $\mathfrak{A}$; we will find it useful to adopt the following suggestive notation:

$$
\text { if } \quad(x, \kappa) \in a, \quad \text { we write } x=d_{\kappa a} .
$$

(Note that in the metalogical interpretation, $d_{\kappa a}$ is the class of the formula $v_{\kappa}=a$.) Now $d_{k a}$ is uniquely determined by $a$ and by $\kappa$ : for if $(x, \kappa) \in a$ and $(y, \kappa) \in a$, then $(x, \kappa) \simeq(y, \kappa)$, so $x=s_{\kappa}^{\kappa} y=y$. From this last statement together with (2.3), it follows that for each term $a \in \mathscr{T}$ and every $\kappa \in$ $\alpha-\Delta a$, there is a unique $x$ in $A$ such that $(x, \kappa) \in a$. Thus,

(2.5) for each $a \in \mathscr{T}$ and $\kappa \in \alpha-\Delta a, d_{\kappa a}$ is uniquely determined by (2.4), and $\left(d_{\kappa a}, \kappa\right) \in a$.

From (2.3) and (2.5) we are able to conclude that

$$
s_{\lambda}^{\kappa} d_{\kappa a}=d_{\lambda a}
$$

for all $a \in \mathscr{T}$ and $\kappa, \lambda \in \alpha-\Delta a$. Furthermore, by (2.1), (2.5) and (2.6),

$$
\begin{aligned}
c_{\kappa} d_{\kappa a} & =1, \quad \text { and } \\
d_{\kappa a} \cdot d_{\mu a} & \leqq d_{\kappa \mu}
\end{aligned}
$$

for all $a \in \mathscr{T}$ and $\kappa, \mu \in \alpha-\Delta a$. Finally, it is worth noting that $d_{\kappa a}$ can be defined for $\kappa \in \Delta a$, too; indeed, we let

$$
d_{\kappa a}=c_{\lambda}\left(d_{\kappa \lambda} \cdot d_{\lambda a}\right)
$$


where $\lambda \in \alpha-(\Delta a \cup\{\kappa\})$. Using [3, 1.5.9(i)] and (2.6), one immediately verifies that (2.9) is independent of the choice of $\lambda$.

For any two terms $a, b \in \mathscr{T}$, we may now define

$$
d_{a b}=c_{\kappa}\left(d_{\kappa a} \cdot d_{\kappa b}\right)
$$

for any $\kappa \in \alpha-(\Delta a \cup \Delta b)$. Because of (2.6) and the fact that $c_{\kappa} x=c_{\lambda} s_{\lambda}^{\kappa} x$ for any $x$ in $A$ and $\lambda \in \alpha-\Delta x[3,1.5 .9(\mathrm{i})], d_{a b}$ does not depend on the choice of $\kappa$. Quite trivially, $d_{a b}=d_{b a}$ for all $a, b \in \mathscr{T}$. We remark that by (2.2) and (2.10), $\Delta d_{a b} \subseteq \Delta a \cup \Delta b$; however, the reverse inclusion does not hold in general.

If $a \in \mathscr{T}$ and $\Delta a=\varnothing$, then $a$ is an algebraic version of a constant. Furthermore, it is worth observing that a variable $v_{\kappa}$ may be intuitively identified with the term $\left(d_{\kappa \lambda}, \lambda\right) / \simeq$, where $\kappa \neq \lambda$.

We conclude this section with a result to be used later.

(2.11) Lemma. For all $a \in \mathscr{T}, \kappa, \lambda \in \alpha-\Delta a$ and $x, y \in A$,

(a) $d_{\kappa a} \cdot d_{\lambda a}=d_{\kappa a} \cdot d_{\kappa \lambda}$,

(b) $c_{\kappa}\left(x \cdot d_{\kappa a}\right) \cdot d_{\lambda a}=c_{\kappa}\left(x \cdot d_{\kappa \lambda}\right) \cdot d_{\lambda a}$ if $\kappa \neq \lambda$,

(c) $c_{\kappa}\left(x \cdot d_{\kappa a}\right) \cdot c_{\kappa}\left(y \cdot d_{\kappa a}\right)=c_{\kappa}\left(x \cdot y \cdot d_{\kappa a}\right)$.

Proof. (a) It follows immediately from (2.8) that $d_{\kappa a} \cdot d_{\lambda a} \leqq d_{\kappa a} \cdot d_{\kappa \lambda}$. But

$$
\begin{aligned}
d_{\kappa a} \cdot d_{\kappa \lambda} & \leqq c_{\kappa}\left(d_{\kappa a} \cdot d_{\kappa \lambda}\right) & & \text { by }\left[3,1.1 .1\left(\mathrm{C}_{2}\right)\right] \\
& =s_{\lambda}^{\kappa} d_{\kappa a} & & \text { by }[3,1.5 .1] \\
& =d_{\lambda a} & & \text { by }(2.6) .
\end{aligned}
$$

By combining the two inequalities we have just established, we get (a).

(b) We are given that $\kappa \neq \lambda$ and $\kappa \notin \Delta a$; so by (2.2) and the fact that $\left(d_{\kappa a}, \kappa\right) \in a$, it follows that $\kappa \notin \Delta d_{\lambda a}$, hence $c_{\kappa} d_{\lambda a}=d_{\lambda a}$. Thus,

$$
\begin{aligned}
c_{\kappa}\left(x \cdot d_{\kappa a}\right) \cdot d_{\lambda a} & =c_{\kappa}\left(x \cdot d_{\kappa a} \cdot d_{\lambda a}\right) & & \text { by }\left[3,1.1 .1\left(\mathrm{C}_{3}\right)\right] \\
& =c_{\kappa}\left(x \cdot d_{\kappa \lambda} \cdot d_{\lambda a}\right) & & \text { by }(\mathrm{a}), \text { above } \\
& =c_{\kappa}\left(x \cdot d_{\kappa \lambda}\right) \cdot d_{\lambda a} & & \text { by }\left[3,1.1 .1\left(\mathrm{C}_{3}\right)\right] .
\end{aligned}
$$

(c) We choose any $\mu<\alpha$ such that $\mu \neq \kappa, \mu \notin \Delta c_{\kappa}\left(x \cdot d_{\kappa a}\right), \mu \notin$ $\Delta c_{\kappa}\left(y \cdot d_{\kappa a}\right)$ and $\mu \notin \Delta c_{\kappa}\left(x \cdot y \cdot d_{\kappa a}\right)$. Then

$$
\begin{aligned}
c_{\kappa}\left(x \cdot y \cdot d_{\kappa a}\right) \cdot d_{\mu a} & =c_{\kappa}\left(x \cdot y \cdot d_{\kappa \mu}\right) \cdot d_{\mu a} & & \text { by (b), above } \\
& =c_{\kappa}\left(x \cdot d_{\kappa \mu}\right) \cdot c_{\kappa}\left(y \cdot d_{\kappa \mu}\right) \cdot d_{\mu a} & & \text { by [3,1.3.6] } \\
& =\left[c_{\kappa}\left(x \cdot d_{\kappa \mu}\right) \cdot d_{\mu a}\right] \cdot\left[c_{\kappa}\left(y \cdot d_{\kappa \mu}\right) \cdot d_{\mu a}\right] & & \\
& =c_{\kappa}\left(x \cdot d_{\kappa a}\right) \cdot c_{\kappa}\left(y \cdot d_{\kappa a}\right) \cdot d_{\mu a} & & \text { by (b), above. }
\end{aligned}
$$

We now apply $c_{\mu}$ to both sides of this equation; by $(2.7),\left[3,1.1 .1\left(\mathrm{C}_{3}\right)\right]$ and our choice of $\mu$, we obtain our result. 
3. Subtituting terms for variables. It is useful to introduce the notion of term-for-variable substitution. If $a$ is a term of $\mathfrak{A}$ and $\kappa<\alpha$, then $s_{a}^{\kappa}$, the operation of a-for-k substitution, is defined as follows: for each $x \in A$,

$$
s_{a}^{\kappa} x=c_{\mu}\left(s_{\mu}^{\kappa} x \cdot d_{\mu a}\right), \quad \text { where } \mu \in \alpha-(\Delta a \cup \Delta x) .
$$

To show that our definition does not depend on the choice of $\mu$, we must show that

$$
c_{\mu}\left(s_{\mu}^{\kappa} x \cdot d_{\mu a}\right)=c_{v}\left(s_{v}^{\kappa} x \cdot d_{v a}\right), \quad \text { for any } \mu, v \in \alpha-(\Delta a \cup \Delta x), \quad \mu \neq v .
$$

The proof is as follows:

$$
\begin{aligned}
c_{\mu}\left(s_{\mu}^{\kappa} x \cdot d_{\mu a}\right) \cdot d_{v a} & =c_{\mu}\left(s_{\mu}^{\kappa} x \cdot d_{\mu v}\right) \cdot d_{v a} & & \text { by }(2.11)(\mathrm{b}) \\
& =s_{v}^{\mu} s_{\mu}^{\kappa} x \cdot d_{v a} & & \text { by }[3,1.5 .1] \\
& =s_{v}^{\kappa} x \cdot d_{v a} & & \text { by }[3,1.5 .11(\mathrm{i})] .
\end{aligned}
$$

Now apply $c_{v}$ to both sides of this equation; as in the proof of (2.11)(b), $\nu \notin \Delta d_{\mu a}$, so by [3,1.6.6., 1.6.8. and 1.6.13], $\nu \notin \Delta c_{\mu}\left(s_{\mu}^{\kappa} x \cdot d_{\mu a}\right)$; our result follows by $\left[3,1.1 .1\left(\mathrm{C}_{3}\right)\right]$ and $(2.7)$.

One easily verifies that the operations $s_{a}^{\kappa}$ are additive; that they are multiplicative follows immediately from $(2.11)(c)$. Furthermore, it is obvious that if $\kappa \notin \Delta a$, then $s_{a}^{\kappa} 0=0$ and $s_{a}^{\kappa} 1=1$. Thus, for each $a \in \mathscr{T}$ and $\kappa<\alpha, s_{a}^{\kappa}$ is a Boolean endomorphism of $\mathfrak{A}$.

\section{BIBLIOGRAPHY}

1. A. Daigneault, Operations in polyadic algebras, Trans. Amer. Math. Soc. 158 (1971), 219-229. MR 44 \#2587.

2. P. R. Halmos, Algebraic logic. III. Predicates, terms and operations in polyadic algebras, Trans. Amer. Math. Soc. 83 (1956), 430-470. MR 19, 113.

3. L. Henkin, D. Monk and A. Tarski, Cylindric algebras. Part I, North-Holland, Amsterdam, 1971.

4. H.-J. Hoehnke, Zur Strukturgleichheit axiomatischer Klassen, Z. Math. Logik Grundlagen Math. 12 (1966), 69-83. MR 32 \#5499.

5. J. R. Shoenfield, Mathematical logic, Addison-Wesley, Reading, Mass., 1967.

Department of Mathematics, Bucknell University, Lewisburg, Pennsylvania 17837 (Current address).

Department of Mathematics, University of California, Berkeley, California 94720 